\title{
El método del estudio de casos en el desarrollo y la evaluación de la competencia transversal instrumental específica de la asignatura Derecho Constitucional Español
}

\section{Cabedo-Mallol, Vicente}

Departamento de Urbanismo. Universitat Politècnica de València. vicamal@upv.es

\begin{abstract}
The case study is a methodology usually used in the learning and teaching of Law. And it's also a very appropriate training activity for the development and evaluation of the specific instrumental transversal competence in legal subjects, as the subject of Spanish Constitutional Law in the Degree in Public Administration and Management. As an instrument to evaluate the level of development of this transversal competence, the use of a rubric is relevant.
\end{abstract}

Keywords: transversal competence, specific instrumental, case study, Law, rubric.

\begin{abstract}
Resumen
El estudio de casos es una metodología utilizada comúnmente en el aprendizaje y enseñanza del Derecho. Y es además una actividad formativa muy apropiada para el desarrollo y evaluación de la competencia transversal instrumental especifica en asignaturas jurídicas, como la asignatura de Derecho constitucional español, que se imparte en el Grado de Gestión y Administración Pública. Como instrumento de evaluación del nivel de desarrollo de dicha competencia transversal, resulta pertinente la utilización de una rúbrica.
\end{abstract}

Palabras clave: competencia transversal, instrumental especifica, estudios de casos, Derecho, rúbrica. 


\section{Introducción}

Las competencias transversales son definidas como habilidades relacionadas con el desarrollo personal, que no dependen de un ámbito temático o disciplinario específico, sino que aparecen en todos los dominios de la actuación profesional y académica (González \& Wangenaar, 2003).

La Univesitat Politècnica de València (UPV) inició en 2014 el "Proyecto Competencias Transversales UPV", cuyo objetivo principal es "acreditar las competencias transversales UPV a los estudiantes egresados en cualquiera de los títulos oficiales impartidos en la Universitat Politècnica de València" (UPV, 2015, p. 2). Estas competencias, que pretenden sintetizar un perfil competencial para todos los alumnos egresados y las alumnas egresadas de la UPV, garantizando que se cubre el marco de referencia de todas las titulaciones, son las siguientes:

CT-01. Comprensión e integración

CT-02. Aplicación y pensamiento práctico

CT-03. Análisis y resolución de problemas

CT-04. Innovación, creatividad y emprendimiento

CT-05. Diseño y proyecto

CT-06. Trabajo en equipo y liderazgo

CT-07. Responsabilidad ética, medioambiental y profesional.

CT-08. Comunicación efectiva

CT-09. Pensamiento crítico

CT-10. Conocimiento de problemas contemporáneos

CT-11. Aprendizaje permanente

CT-12. Planificación y gestión del tiempo

CT-13. Instrumental específica

A efectos prácticos, a partir del curso 2015-2016, en la guía docente de cada asignatura debe indicarse, con relación al listado anterior de competencias, cuáles no se trabajan, cuáles se trabajan pero no se evalúan, y cuáles son "punto de control". En este último caso, dado que se va a evaluar la competencia correspondiente, en la aplicación de la guía aparece un desplegable en el que se indica:

- Un listado de actividades a realizar en la asignatura.

- Una concreción de esas actividades dentro del desarrollo de la asignatura.

- La manera en la que se van a evaluar.

En este trabajo centraré la atención en la competencia transversal instrumental específica (CT-13) y en su evaluación, dado que, a partir del curso 2017-2018, se estableció que la 
asignatura de Derecho constitucional español, del Grado de Gestión y Administración Pública (GAP), fuese punto de control de la indicada competencia transversal. Ser punto de control implica plantear actividades para, en el desarrollo de los contenidos, trabajar la competencia transversal y evaluarla, recogiendo evidencias de los logros alcanzados. En estas circunstancias, consideré oportuno, como responsable de la referida asignatura, desarrollar y evaluar la competencia transversal instrumental específica recurriendo al método del estudio de casos.

\section{Las metodologías activas y el estudio de casos}

Las metodologías activas de aprendizaje nacieron como una alternativa a la tradicional clase magistral. Ello no quiere decir que deba erradicarse el método tradicional como sistema de enseñanza-aprendizaje, en general, y en el Derecho, en particular. Se trata de adoptarlo o ajustarlo a las necesidades actuales al complementarlo con otros métodos.

Entre las metodologías activas más recurrentes encontramos las siguientes: el método del caso; el aprendizaje basado en problemas; la discusión controversial; el aprendizaje cooperativo; el contrato de aprendizaje; el aprendizaje basado en proyectos; la técnica expositiva; y la simulación y el juego.

De los citados métodos activos, es el método de casos, sin duda alguna, el más utilizado en el aprendizaje del Derecho. No estamos, en este caso, ante una nueva metodología, dado que su origen se remonta a finales del s. XIX. Es a partir de 1870 cuando, en la universidad de Harvard, se introdujo este enfoque, que en ese momento sí constituyó toda una novedad en el estudio del Derecho. Con el estudio y análisis de fallos jurisprudenciales se preparaba a la o estudiante para el desarrollo de la práctica judicial.

El método de casos propiciaría una educación formativa e informativa, teórico- práctica y activa- participativa. El mismo, de acuerdo con Sosa y Silva García, Lacavex Berunen y Rodríguez Cebrero (2011), presentaría las siguientes características:

a) Es interactivo y dinámico.

b) Está centrado en el alumno.

c) Se basa en una situación real.

d) El profesor actúa como facilitador del proceso, orientando la discusión.

e) Es un enlace entre la teoría y la práctica.

Es fundamental que el profesor o la profesora se asegure, eso sí, de que los alumnos y las alumnas cuenten con una buena base teórica que les permita trabajar con el caso y transferir sus conocimientos a una situación real. Y, por supuesto, también corresponde al o la docente explicarles previamente cómo se resuelve un caso práctico. A estos efectos, desde los inicios de los estudios de GAP en la UPV, los profesores del área de Derecho constitucional hemos venido utilizado el manual "Prácticas de Derecho Constitucional" (1992), del Dr. Antonio Colomer y el Dr. José Luis López González. El mismo distingue en el proceso de resolución de un caso las siguientes cinco fases: 
I. Fase de análisis comprensivo del texto propuesto.

II. Fase de estudio de los problemas jurídicos que plantea el caso.

III. Fase de localización de las normas jurídicas aplicables al supuesto de hecho.

IV. Fase de análisis de los problemas jurídicos y aplicación de normas.

V. Fase de conclusiones.

Tras la explicación de las indicadas fases, se facilita al alumnado, por su claridad, el supuesto práctico resuelto que se recoge en el referido manual.

Y como casos a resolver por los alumnos y las alumnas de la asignatura de Derecho constitucional español, selecciono dos Sentencias del Tribunal Supremo, que serán objeto de análisis tras el estudio de la unidad didáctica relativa a los derechos fundamentales.

Conviene, por último, no olvidar, a la hora de evaluar dichas actividades, que no estamos en presencia de estudiantes de Derecho, por lo que no tienen como objetivo prepararse para la práctica forense.

Son estas, pues, entre otras, actividades que se proponen para la enseñanza-aprendizaje de la asignatura de Derecho constitucional, pero que, al mismo tiempo, como se expondrá a continuación, permiten desarrollar la adquisición de la competencia instrumental específica.

\section{La evaluación de la competencia transversal instrumental específica en la asignatura de Derecho constitucional español.}

La competencia instrumental específica, de acuerdo con el documento Proyecto Competencias Transversales UPV (2015), hace referencia a saber utilizar adecuadamente, por parte del alumno y la alumna, las herramientas actualizadas y tecnologías necesarias para la práctica de la profesión. El o la estudiante será, por tanto, capaz de identificar las herramientas más adecuadas en cada caso, conociendo sus utilidades y siendo capaz de integrarlas y combinarlas para poder resolver un problema, realizar un proyecto o un experimento.

Desde el Instituto de Ciencias de la Educación de la UPV se han establecido tres niveles de dominio para detectar avances en el desarrollo de la competencia. A nuestros efectos nos interesa el primer nivel, dado que muestra los resultados de aprendizaje que sería deseable que los y las estudiantes hubiesen alcanzado al finalizar el segundo curso de grado. En concreto, el resultado a alcanzar consistiría en emplear correctamente las herramientas básicas del ámbito profesional de forma guiada. Veamos a continuación (Tabla 1) la rúbrica (matriz de evaluación) de dicho primer nivel, con tres indicadores de los aspectos a analizar: 1) Identificar las herramientas básicas y su utilidad; 2) Manejar las herramientas básicas siguiendo unas instrucciones previamente dadas; y 3) Hacer uso seguro y responsable de las herramientas. 
Tabla 1. Rúbrica competencia transversal instrumental específica (primer nivel) RÚBRICA UPV CT-13. INSTRUMENTAL ESPECÍFICA

Nivel de dominio 1

Resultado de aprendizaje: emplear correctamente las herramientas básicas del ámbito profesional de forma guiada.

\begin{tabular}{|c|c|c|c|c|}
\hline \multirow{2}{*}{ INDICADORES } & \multicolumn{4}{|c|}{ DESCRIPTORES } \\
\hline & D. No alcanzado & C. En desarrollo & B. Bien /adecuado & A. Excelente/ejemplar \\
\hline $\begin{array}{l}\text { Identifica las } \\
\text { herramientas básicas y } \\
\text { su utilidad }\end{array}$ & $\begin{array}{l}\text { No identifica las } \\
\text { herramientas básicas }\end{array}$ & $\begin{array}{l}\text { Identifica las herramientas } \\
\text { básicos pero no } \\
\text { reconoce su Utlilidad }\end{array}$ & $\begin{array}{c}\text { Identifica las heramientas } \\
\text { básicos y su función } \\
\text { principal }\end{array}$ & $\begin{array}{l}\text { Identifica funciones } \\
\text { adicionales de las } \\
\text { herramientas }\end{array}$ \\
\hline $\begin{array}{l}\text { Maneja las } \\
\text { herramientas básicas } \\
\text { siguiendo unas } \\
\text { instrucciones } \\
\text { previamente dadas }\end{array}$ & $\begin{array}{l}\text { No es capaz de seguir } \\
\text { unas instrucciones }\end{array}$ & $\begin{array}{l}\text { Maneja las herramientas } \\
\text { siguiendo las instrucciones } \\
\text { pero sin comprender lo } \\
\text { que está haciendo }\end{array}$ & $\begin{array}{c}\text { Maneja las herramientas } \\
\text { siguiendo las instrucciones } \\
\text { y comprendiendo lo que } \\
\text { está haciendo }\end{array}$ & $\begin{array}{c}\text { Maneja las herramientas } \\
\text { con solturae } \\
\text { interpretando los } \\
\text { resultodos obteridos }\end{array}$ \\
\hline $\begin{array}{l}\text { Hace uso seguro y } \\
\text { responsable de las } \\
\text { herramientas }\end{array}$ & $\begin{array}{l}\text { No sigue las } \\
\text { recomendaciones de } \\
\text { seguridad }\end{array}$ & $\begin{array}{l}\text { Identifica los riesgos y los } \\
\text { elementos de seguridad } \\
\text { disporibles }\end{array}$ & $\begin{array}{c}\text { Hace un uso seguro de las } \\
\text { herramientas }\end{array}$ & $\begin{array}{l}\text { Hace un uso cuidadoso } \\
\text { de las herramientas. } \\
\text { minimizando los riesgos } \\
\text { para las personas y para } \\
\text { los equipos. }\end{array}$ \\
\hline
\end{tabular}

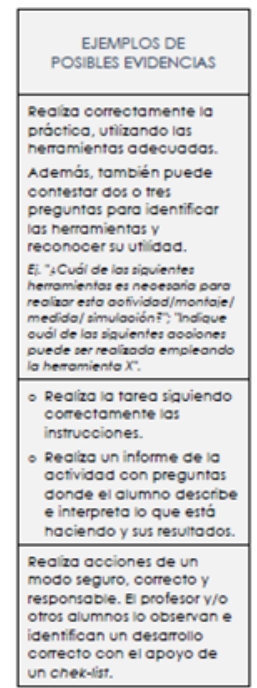

Fuente: Bonet et al. (2015, p. 98)

Para evaluar esta competencia transversal en la asignatura de Derecho constitucional español propuse, como he señalado anteriormente, el estudio de casos, dado que esta metodología comporta la necesidad de utilizar las herramientas necesarias y tecnologías para el ejercicio práctico de la profesión de jurista. Profesión que requiere ineludiblemente el conocimiento y manejo de las fuentes del Derecho y de la jurisprudencia. En este sentido, la utilización de las bases de datos legislativas y jurisprudenciales son esenciales para la búsqueda de la legislación y, en su caso, de la jurisprudencia pertinente para la resolución de los casos prácticos. Los alumnos y las alumnas deben, por tanto, familiarizarse con los métodos de búsqueda y el funcionamiento de buscadores y bases de datos jurídicas.

En definitiva, como punto de control de la competencia instrumental específica, se evalúa el aprendizaje del alumnado en la identificación, el manejo y uso seguro y responsable, por una parte, de bases de datos y buscadores gratuitos de legislación, tanto de España como de la Unión Europea, como son la base de datos del portal Noticias Jurídicas, el buscador de legislación del Boletín Oficial del Estado, o la Base de datos de Eurlex; y, por otra, de bases de datos y buscadores de jurisprudencia también gratuitos como la Base de datos del Centro de Documentación Judicial (CENDOJ) del Consejo General del Poder Judicial o el buscador de jurisprudencia del Tribunal Constitucional.

Establecido el método para la adquisición de la competencia instrumental específica, queda por determinar las herramientas para evaluar los resultados del aprendizaje y puntuar los mismos. Entre dichas herramientas, destacan las denominadas rúbricas. En este sentido, las mismas son consideradas "una herramienta de puntuación que enumera 
los criterios que deben tenerse en cuenta para la elaboración de un trabajo escrito", y que "además articula las gradaciones de calidad para cada uno de estos criterios, desde sobresaliente hasta deficiente" (Andrade, 2010, p. 158). Una definición más precisa señalará que son "guías de puntuación usadas en la evaluación del desempeño de los estudiantes que describen las características específicas de un producto, proyecto o tarea en varios niveles de rendimiento, con el fin de clarificar lo que se espera del trabajo del alumno, de valorar su ejecución y de facilitar la proporción de feedback" (Andrade, 2005; Mertler, 2001; citados en Fernández, 2010, p. 24).

Existen muchos ejemplos de rúbricas dependiendo de la técnica de evaluación empleada, incluso existen páginas web que ayudan a crearlas (v.gr. web rubistar: http://rubistar.4teachers.org/index.php). En mi caso, me es de utilidad la rúbrica anteriormente expuesta elaborada por Bonet et al. (2015). Esta rúbrica les es explicada a los alumnos y a las alumnas al inicio del curso, dado que deben ser sabedores de cómo se les vas evaluar el aprendizaje de la competencia transversal.

\section{Conclusiones}

La competencia transversal instrumental específica ha sido punto de control en la asignatura de Derecho Constitucional Español durante los cursos académicos 2017-2018 y 2018-2019. Las actividades formativas llevadas a cabo para el aprendizaje de esta competencia se han centrado en el estudio de casos. Esta metodología, utilizada comúnmente en el aprendizaje y enseñanza del Derecho, ha resultado satisfactoria para el desarrollo y evaluación de la referida competencia transversal.

El estudio de casos jurídicos requiere, como se ha indicado, de una fase, entre otras, de localización de las normas jurídicas y, en su caso, de la jurisprudencia aplicables al supuesto de hecho del caso en cuestión. Para ello, el alumno y la alumna puede recurrir a la red de redes (internet), pero es de vital importancia que previamente conozca los buscadores que debe utilizar para encontrar tanto la legislación como la jurisprudencia aplicables. Además, el y la estudiante debe aprender a manejar estos buscadores. De ahí que el método de estudio de casos sea tan apropiado para la adquisición de la competencia instrumental específica en una asignatura jurídica.

La metodología de estudio de casos es también apropiada para el desarrollo de otras competencias transversales como la de pensamiento crítico (CT-09) o la de análisis y resolución de problemas (CT-03). En este sentido, esta última competencia se trabaja, con la indicada metodología, desde el curso 2017-2018, en la asignatura Inmigrantes y Emigrantes, de la que soy el profesor responsable.

Por último, por lo que respecta a la evaluación de la competencia transversal instrumental específica o la de análisis y resolución de problemas, la utilización de una rúbrica presenta una serie de ventajas como la objetividad, dado que los criterios de medición están explicitados y son conocidos por los alumnos y las alumnas. 


\section{Bibliografía}

ANDRADE, H. G. (2005). "Teaching with rubrics" en College Teaching, vol. 53, $\mathrm{n}^{\mathrm{o}}$ 1, p. 27-30.

BOLETÍN OFICIAL DEL ESTADO. Legislación. Búsqueda sencilla. $<$ https://www.boe.es/buscar/legislacion.php> [Consulta: 19 de junio de 2019].

COLOMER VIADEL, A. Y LOPEZ GONZALEZ, J. L. (1992). Prácticas de Derecho Constitucional. València: Tirant lo Blanch.

CONSEJO GENERAL DEL PODER JUDICIAL. Centro de Documentación Judicial. $<$ http://www.poderjudicial.es/search/indexAN.jsp> [Consulta: 19 de junio de 2019].

EUR-Lex. El acceso al Derecho de la Unión Europea. <http://eurlex.europa.eu/homepage.html?locale $=$ es $>$ [Consulta: 19 de junio de 2019].

FERNANDEZ MARCH, A. (2010). "La evaluación orientada al aprendizaje en un modelo de formación por competencias en la educación universitaria" en Revista de Docencia Universitaria, vol. $8, \mathrm{n}^{\circ} 1,11-34$

GONZÁLEZ, C. Y WANGENAAR, R. (2003). Tuning educational structures in Europe. Bilbao: Universidad de Deusto.

NOTICIAS JURÍDICAS. Buscador de legilación. $<$ http://noticias.juridicas.com/base_datos $>$ [Consulta: 19 de junio de 2019].

SOSA Y SILVA GARCIA, Y., LACAVEX BERUNEN, M. A. Y RODRIGUEZ CEBRERO, J. (2011). "La enseñanza del derecho a través del estudio de casos" en Alegatos, vol. 79, p. 825-846.

TRIBUNAL CONSTITUCIONAL. Buscdor de jurisprudencia constitucional. $<$ http://hj.tribunalconstitucional.es/> [Consulta: 30 de junio de 2017].

UNIVERSITAT POLITECNICA DE VALENCIA (2014). Proyecto competencias transversales UPV. València: UPV. $<$ https://www.upv.es/entidades/ICE/info/U0724624.pdf $>$ [Consulta: 30 de junio de 2017]. 